\title{
Bulk-mediated diffusion on a planar surface: full solution
}

\author{
Aleksei V. Chechkin, ${ }^{1,2}$ Irwin M. Zaid,${ }^{3}$ Michael A. Lomholt ${ }^{4}$ Igor M. Sokolov, ${ }^{5}$ and Ralf Metzler ${ }^{2,6}$ \\ ${ }^{1}$ Institute for Theoretical Physics NSC KIPT, Akademicheskaya st.1, 61108 Kharkov, Ukraine \\ ${ }^{2}$ Institute for Physics 85 Astronomy, University of Potsdam, D-14476 Potsdam-Golm, Germany \\ ${ }^{3}$ Rudolf Peierls Centre for Theoretical Physics, University of Oxford, \\ 1 Keble Road, Oxford OX1 3NP, United Kingdom \\ ${ }^{4}$ MEMPHYS - Center for Biomembrane Physics, \\ Department of Physics, Chemistry, and Pharmacy, \\ University of Southern Denmark, Campusvej 55, 5230 Odense M, Denmark \\ ${ }^{5}$ Institut für Physik, Humboldt Universität zu Berlin, Newtonstraße 15, 12489 Berlin, FRG \\ ${ }^{6}$ Department of Physics, Technical University of Tampere, FI-33101 Tampere, Finland
}

(Dated: 3rd November 2018)

\begin{abstract}
We consider the effective surface motion of a particle that intermittently unbinds from a planar surface and performs bulk excursions. Based on a random walk approach we derive the diffusion equations for surface and bulk diffusion including the surface-bulk coupling. From these exact dynamic equations we analytically obtain the propagator of the effective surface motion. This approach allows us to deduce a superdiffusive, Cauchy-type behavior on the surface, together with exact cutoffs limiting the Cauchy form. Moreover we study the long-time dynamics for the surface motion.
\end{abstract}

PACS numbers: 05.40.Fb,02.50.Ey,82.20.-w,87.16.-b

\section{INTRODUCTION}

Interfaces and the interaction of particles with them play a crucial role on small scales in biology and technology. For instance, biopolymers such as proteins or enzymes diffusing in biological cells intermittently bind to cellular membranes, or individual bacteria forming a biofilm on a surface use bulk excursions to efficiently relocate. Similarly the exchange between a liquid phase with a solid surface is an important phenomenon in the selfassembly of surface layer films and is a ubiquitous process in emulsions. This bulk-mediated surface diffusion, schematically shown in Fig. 1, was previously analyzed in terms of scaling arguments and simulations [1-4] , and was unveiled in field cycling NMR experiments in porous glasses [5]. Moreover, effects of bulk-surface interchange were reported on proton transport across biological membranes [6]. Recent studies are concerned with effects of bulk-surface exchange on reaction rates in interfacial systems [7] and with surface diffusion of coppper atoms in nanowire fabrication [8].

The remarkable finding of the bulk mediated surface diffusion model is that the effective surface motion is characterized by a Cauchy propagator [1-4]

$$
n_{s}(\mathbf{r}, t) \simeq \frac{c^{1 / 2} t}{2 \pi\left(r^{2}+c t^{2}\right)^{3 / 2}},
$$

where $\simeq$ denotes a scaling property ignoring multiplicative constants, and $c$ is a dimensional factor. The associated stochastic transport is of superdiffusive nature [1 5, 9],

$$
\left\langle\mathbf{r}^{2}(t)\right\rangle_{s} \simeq t^{3 / 2}
$$

Here we present a strictly analytical approach to this process. Our findings corroborate the previous scaling

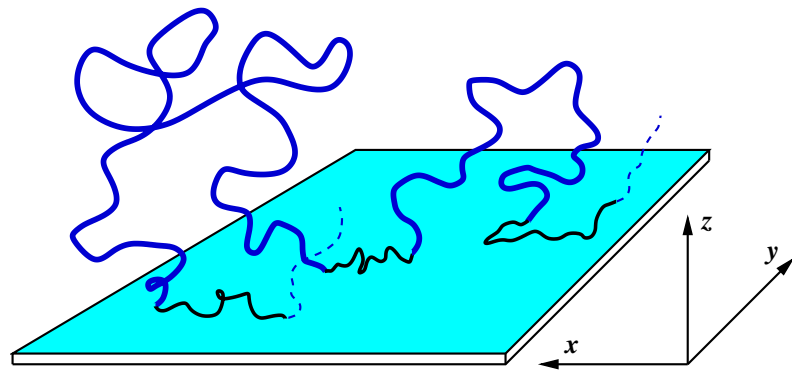

Figure 1: Schematic of the bulk mediated surface diffusion: The thinner (black) lines show the motion along the planar surface with surface diffusivity $D_{s}$. Following dissociation from the surface, thicker (blue) lines depict excursions into the bulk volume, in which the diffusion constant is $D_{b}$. Eventually, the particle rebinds to the surface.

results for superdiffusion, however, we also derive the cutoffs to this behavior: at sufficiently long distances, the Cauchy propagator turns over to a Gaussian wing. Moreover at longer times the effective surface diffusion becomes subdiffusive, due to the fact that the particle spends less and less time on the surface. Normalized to the time-dependent surface coverage the effective surface diffusion turns over from superdiffusion to normal diffusion.

\section{DERIVATION FROM A DISCRETE RANDOM WALK PROCESS}

We start with a derivation of the coupling between surface and bulk in a discrete random walk process along the $z$ coordinate perpendicular to the surface (Fig. 11). Let $N_{i}$ with $i=1,2, \ldots$ denote the number of particles at 
site $i$ of this one-dimensional lattice with spacing $a$. The number of particles on the surface at lattice site $i=0$ are termed $\mathcal{N}_{0}$. The exchange of particles is possible only via nearest neighbor jumps. Each jump event between bulk sites $i=1,2, \ldots$ is associated with the typical waiting time $\tau$. For the exchange between the surface and site $i=1$ we then have the following law

$$
\frac{d \mathcal{N}_{0}(t)}{d t}=\frac{1}{2 \tau} N_{1}-\frac{1}{\tau_{\mathrm{des}}} \mathcal{N}_{0},
$$

where $\tau_{\text {des }}$ is the characteristic time for desorption from the surface. The probability of adsorption to the surface here is one, i.e., a particle adds to the surface population automatically when moving from site 1 to site 0 . The exchange site at $i=1$ between the surface at $i=0$ and the next bulk site $i=2$ is governed by the balance relation

$$
\frac{d N_{1}(t)}{d t}=\frac{1}{\tau_{\text {des }}} \mathcal{N}_{0}-\frac{1}{\tau} N_{1}+\frac{1}{2 \tau} N_{2} .
$$

Finally, the bulk sites $i=2,3, \ldots$ are governed by equations of the form

$$
\frac{d N_{2}(t)}{d t}=\frac{1}{2 \tau} N_{1}+\frac{1}{2 \tau} N_{3}-\frac{1}{\tau} N_{2},
$$

etc. Let us define the number of "bulk" particles at the surface site $i=0$ through

$$
N_{0} \equiv \frac{2 \tau}{\tau_{\text {des }}} \mathcal{N}_{0}
$$

This trick allows us to formulate the exchange equation also for site $i=1$ in a homogeneous form. Namely, from Eq. (3) we have

$$
\frac{d \mathcal{N}_{0}(t)}{d t}=\frac{1}{2 \tau}\left(N_{1}-N_{0}\right)
$$

Moreover, from Eqs. (5) we find

$$
\frac{d N_{i}(t)}{d t}=\frac{1}{2 \tau}\left(N_{i-1}-2 N_{i}+N_{i+1}\right),
$$

for $i \geq 1$.

Let us now take the continuum limit. For that purpose we make a transition from $\mathcal{N}_{0} \rightarrow n_{s}$ as the number of surface particles, and $N_{i} \rightarrow a n_{b}$ for the bulk concentration of particles. The factor $a$ may be viewed as the lattice constant measuring the distance between successive sites along the $z$ axis. As the bulk density is a function of $z$ while the surface particles are all assembled at $z=0$, we need this length scale $a$ to match dimensionalities between $n_{s}$ and $n_{b}$ which are $\left[n_{s}\right]=\mathrm{cm}^{-2}$ and $\left[n_{b}\right]=\mathrm{cm}^{-3}$. Expansion of the right hand side of Eq. (7) yields the surface-bulk coupling

$$
\frac{\partial n_{s}(t)}{\partial t}=\left.\frac{a}{2 \tau} a \frac{\partial n_{b}(z, t)}{\partial z}\right|_{z=0}
$$

Similarly from Eq. (8) we obtain the bulk diffusion equation

$$
\frac{\partial n_{b}(z, t)}{\partial t}=\frac{1}{2 \tau} a^{2} \frac{\partial^{2} n_{b}}{\partial z^{2}}
$$

Finally, the boundary condition

$$
\left.\frac{a}{2 \tau} n_{b}(z, t)\right|_{z=0}=\frac{1}{\tau_{\mathrm{des}}} n_{s}
$$

stems from our definition (6). This completes the description of the particle exchange between surface and bulk, as well as the diffusion of bulk particles along the $z$ axis.

We now turn back to the full three-dimensional problem and add to above equations the $(x, y)$ directions (see Fig. 11). As the motion in the perpendicular $z$ direction is fully independent, we may simply adjust the Laplacian to three dimensions, ending up with the diffusion equation

$$
\frac{\partial n_{b}(x, y, z, t)}{\partial t}=D_{b}\left(\frac{\partial^{2}}{\partial x^{2}}+\frac{\partial^{2}}{\partial y^{2}}+\frac{\partial^{2}}{\partial z^{2}}\right) .
$$

with diffusivity $D_{b}$. For the surface diffusion along the $x$ and $y$ coordinates with $z=0$, we end up with the twodimensional diffusion equation with diffusivity $D_{s}$, plus the bulk-surface exchange term:

$$
\frac{\partial n_{s}(x, y, t)}{\partial t}=D_{s}\left(\frac{\partial^{2}}{\partial x^{2}}+\frac{\partial^{2}}{\partial y^{2}}\right) n_{s}+\left.D_{b} \frac{\partial n_{b}}{\partial z}\right|_{z=0}
$$

These two equations are valid in the range $0 \leq z<\infty$ and $-\infty<x, y<\infty$, and are supplemented by the initial condition

$$
\left.n_{s}(x, y, t)\right|_{t=0}=n_{0} \delta(x) \delta(y)
$$

indicating that initially the particles are all concentrated on the surface at $x=y=0$. Moreover we observe the boundary condition for $n_{b}$,

$$
\left.n_{b}(x, y, z, t)\right|_{z=0}=\mu n_{s}(x, y, t)
$$

and the bulk initial condition

$$
\left.n_{b}(x, y, z, t)\right|_{t=0}=0
$$

In what follows, for simplicity of notation we use a unit initial concentration $n_{0}$.

The surface and bulk diffusivities can be expressed in terms of the lattice constant $a$ and the typical waiting times between jumps in the bulk, $\tau$, and on the surface, $\tau_{s}$, through

$$
D_{b} \equiv \frac{a^{2}}{6 \tau}
$$

and

$$
D_{s} \equiv \frac{a^{2}}{4 \tau_{s}}
$$


respectively. In the continuum limit, both $a$ and $\tau$ (or $\tau_{s}$ ) tend to zero such that the diffusion constants remain finite. In many physical systems bulk diffusion is considerably faster, such that $D_{s} \ll D_{b}$. Above we also introduced the coupling parameter

$$
\mu \equiv \frac{a}{D_{b} \tau_{\text {des }}}
$$

of physical dimension $[\mu]=1 / \mathrm{cm}$. Small values of $\mu$ at fixed $a$ and $D_{b}$ correspond to slow bulk-surface exchange.

For consistency we derive the overall number of particles. To this end we define the number particles on the surface, $N_{s}(t)$, and in the bulk, $N_{b}(t)$ through the relations

$$
N_{s}(t)=\int_{-\infty}^{\infty} d x \int_{-\infty}^{\infty} d y n_{s}(x, y, t)
$$

and

$$
N_{b}(t)=\int_{0}^{\infty} d z \int_{-\infty}^{\infty} d x \int_{-\infty}^{\infty} d y n_{s}(x, y, t)
$$

From integration of Eq. (13) we find

$$
\frac{d N_{s}(t)}{d t}=\left.D_{b} \frac{\partial}{\partial z} \int_{-\infty}^{\infty} d x \int_{-\infty}^{\infty} d y n_{b}(x, y, z, t)\right|_{z=0}
$$

Similarly, Eq. (12) yields

$$
\begin{aligned}
\frac{d N_{b}(t)}{d t} & =D_{b} \int_{0}^{\infty} d z \frac{\partial^{2}}{\partial z^{2}} \int_{-\infty}^{\infty} d x \int_{-\infty}^{\infty} d y n_{b}(x, y, z, t) \\
& =-\left.D_{b} \frac{\partial}{\partial z} \int_{-\infty}^{\infty} d x \int_{-\infty}^{\infty} d y n_{b}(x, y, z, t)\right|_{z=0}(23)
\end{aligned}
$$

Combination of Eqs. (22) and (23) produces

$$
\frac{d}{d t}\left(N_{s}(t)+N_{b}(t)\right)=0
$$

Thus the overall number of particles is conserved, as it should be.

\section{SOLUTION OF THE COUPLED DIFFUSION PROBLEM}

To solve the set of coupled equations (13) and (12) for the specified initial and boundary value problem, we start by defining the two-dimensional Green's function

$$
G_{s}\left(x, x^{\prime}, y, y^{\prime}, t\right) \equiv G_{s}\left(x-x^{\prime}, y-y^{\prime}, t\right)=\frac{1}{\sqrt{4 \pi D_{s} t}} \exp \left(-\frac{\left(x-x^{\prime}\right)^{2}+\left(y-y^{\prime}\right)^{2}}{4 D_{s} t}\right) .
$$

Then, the solution of Eq. (13) becomes

$$
\begin{aligned}
n_{s}(x, y, t)= & \int d x^{\prime} \int d y^{\prime} G_{s}\left(x, x^{\prime}, y, y^{\prime}, t\right) n_{s}\left(x^{\prime}, y^{\prime}, t=0\right) \\
& +\int_{0}^{t} d t^{\prime} \int d x^{\prime} \int d y^{\prime} G_{s}\left(x, x^{\prime}, y, y^{\prime}, t-t^{\prime}\right)\left(D_{b} \frac{\partial n_{b}\left(x^{\prime}, y^{\prime}, z, t^{\prime}\right)}{\partial z}\right)_{z=0} .
\end{aligned}
$$

With initial condition (14) we thus find

$$
n_{s}\left(k_{x}, k_{y}, s\right)=G_{s}\left(k_{x}, k_{y}, s\right)+G_{s}\left(k_{x}, k_{y}, s\right) \mathscr{L}\left\{\mathscr{F}\left\{D_{b}\left(\frac{\partial n_{b}(x, y, z, t)}{\partial z}\right)_{z=0} ; x \rightarrow k_{x} ; y \rightarrow k_{y}\right\} ; t \rightarrow s\right\}
$$

where

$$
G_{s}\left(k_{x}, k_{y}, s\right)=\frac{1}{s+D_{b}\left[k_{x}^{2}+k_{y}^{2}\right]}
$$

is the Fourier-Laplace transform of the surface Green's function $G_{s}$. Here and in the following we denote the Laplace and Fourier transform of a function by explicit dependence on the image variables, that is,

$$
f(s)=\mathscr{L}\{f(t) ; t \rightarrow s\}=\int_{0}^{\infty} f(t) e^{-s t} d t \quad \text { and } \quad g(k)=\mathscr{F}\{g(x) ; x \rightarrow k\}=\int_{-\infty}^{\infty} g(x) e^{i k x} d x
$$

The bulk particle density according to Eq. (12) is given by the formal expression [11]

$$
n_{b}(x, y, z, t)=\frac{z}{\left(4 \pi D_{b}\right)^{3 / 2}} \int_{0}^{t} d t^{\prime} \int d x^{\prime} \int d y^{\prime} \frac{\mu n_{s}\left(x^{\prime}, y^{\prime}, t^{\prime}\right)}{\left(t-t^{\prime}\right)^{5 / 2}} \exp \left(-\frac{\left(x-x^{\prime}\right)^{2}+\left(y-y^{\prime}\right)^{2}+z^{2}}{4 D_{b}\left(t-t^{\prime}\right)}\right)
$$


From this expression one can indeed show that, despite the factor $z$, the coupling equation (15) is fulfilled, see Appendix B. Now, Eq. (27) requires the derivative of expression (30) with respect to $z$, evaluated at $z=0$. To find that expression, we first differentiate

$$
\frac{\partial n_{b}}{\partial z}=\frac{\mu}{\left(4 \pi D_{b}\right)^{3 / 2}} \int_{0}^{t} d t^{\prime} \int d x^{\prime} \int d y^{\prime} \frac{n_{s}\left(x^{\prime}, y^{\prime}, t^{\prime}\right)}{\left(t-t^{\prime}\right)^{5 / 2}} \exp \left(-\frac{\left(x-x^{\prime}\right)^{2}+\left(y-y^{\prime}\right)^{2}+z^{2}}{4 D_{b}\left(t-t^{\prime}\right)}\right)\left[1-\frac{z^{2}}{2 D_{b}\left(t-t^{\prime}\right)}\right]
$$

and then calculate its Fourier-Laplace transform

$$
\mathscr{L}\left\{\mathscr{F}\left\{D_{b}\left(\frac{\partial n_{b}}{\partial z}\right)_{z=0}\right\}\right\}=\frac{\mu D_{b}}{\sqrt{4 \pi D_{b}}} n_{s}\left(k_{x}, k_{y}, s\right) \mathscr{L}\left\{\frac{\exp \left(-D_{b}\left[k_{x}^{2}+k_{y}^{2}\right] t\right)}{t^{3 / 2}}\right\}_{z=0},
$$

due to the convolution nature of expression (31). The Laplace transform is evaluated by help of the shift theorem, yielding

$$
\mathscr{L}\left\{\frac{\exp \left(-D_{b}\left[k_{x}^{2}+k_{y}^{2}\right] t\right)}{t^{3 / 2}}\right\}=\mathscr{L}\left\{\frac{1}{t^{3 / 2}}\right\}_{s \rightarrow s+D_{b}\left[k_{x}^{2}+k_{y}^{2}\right]}=-\sqrt{4 \pi\left(s+D_{b}\left[k_{x}^{2}+k_{y}^{2}\right]\right)} .
$$

We thus finally obtain

$$
\mathscr{L}\left\{\mathscr{F}\left\{D_{b}\left(\frac{\partial n_{b}}{\partial z}\right)_{z=0}\right\}\right\}=-\mu D_{b}^{1 / 2} \sqrt{s+D_{b}\left(k_{x}^{2}+k_{y}^{2}\right)} n_{s}\left(k_{x}, k_{y}, s\right) .
$$

Insertion of this equation into expression (27) delivers the solution for the surface density in Fourier-Laplace space,

$n_{s}\left(k_{x}, k_{y}, s\right)=\frac{G_{s}\left(k_{x}, k_{y}, s\right)}{1+\mu D_{b}^{1 / 2} G_{s}\left(k_{x}, k_{y}, s\right) \sqrt{s+D_{b}\left[k_{x}^{2}+k_{y}^{2}\right]}}$,

where the Fourier-Laplace transform of the Green's function $G_{s}$ was defined in Eq. (28). After some transformations we arrive at the exact closed form expression

$$
n_{s}\left(k_{x}, k_{y}, s\right)=\frac{1}{s+D_{s}\left[k_{x}^{2}+k_{y}^{2}\right]+\chi \sqrt{s+D_{b}\left[k_{x}^{2}+k_{y}^{2}\right]}}
$$

with the rescaled coupling parameter

$$
\chi \equiv \mu D_{b}^{1 / 2}=\frac{a}{D_{b}^{1 / 2} \tau_{\text {des }}}
$$

of dimension $[\chi]=1 / \sec ^{1 / 2}$. Relation (36) is the main result of this work, and we now consider the consequences to the surface motion effected by the bulk mediation.

\section{EFFECTIVE SURFACE BEHAVIOR}

We first determine the number of particles on the surface,

$$
N_{s}(t)=\int_{-\infty}^{\infty} \int_{-\infty}^{\infty} n_{s}(x, y, t) d x d y
$$

whose Laplace transform is

$$
N_{s}(s)=\left.n\left(k_{x}, k_{y}, s\right)\right|_{k_{x}=k_{y}=0}=\frac{1}{s+\chi s^{1 / 2}} .
$$

Inverse Laplace transformation then yields the exact expression

$$
N_{s}(t)=e^{\chi^{2} t} \operatorname{erfc}\left(\chi t^{1 / 2}\right)
$$

where we use the complementary error function

$$
\operatorname{erfc}(z)=\frac{2}{\sqrt{\pi}} \int_{z}^{\infty} e^{-\xi^{2}} d \xi=1-\operatorname{erf}(z)
$$

At short times $t \ll \chi^{-2}$, this leads to the initial decay

$$
N_{s}(t) \sim 1-\frac{2 \chi}{\sqrt{\pi}} t^{1 / 2}
$$

of the number of surface particles, eventually turning into the long time behavior

$$
N_{s}(t) \sim \frac{1}{\chi \sqrt{\pi t}}
$$

The asymptotic $1 / \sqrt{\pi t}$ decay stems from the returning dynamics to the origin $z=0$ of a Brownian motion along the $z$ coordinate, i.e., it is proportional to the normalization factor of a one-dimensional Brownian motion. The additional prefactor $\chi$ rescales time with respect to the efficiency of the surface-bulk exchange.

We now turn to the surface dynamics, as quantified by the effective mean squared displacement along the sur- 
face. In the Laplace domain,

$$
\begin{aligned}
\left\langle\mathbf{r}^{2}(s)\right\rangle_{s} & =-\left.\nabla_{k_{x}, k_{y}}^{2} n\left(k_{x}, k_{y}, s\right)\right|_{k_{x}=k_{y}=0} \\
& =-\left.\left[\frac{\partial^{2}}{\partial k^{2}}+\frac{1}{k} \frac{\partial}{\partial k}\right] n\left(k_{x}, k_{y}, s\right)\right|_{k_{x}=k_{y}=0} \\
& =\frac{4 D_{s}}{s(\sqrt{s}+\chi)^{2}}+\frac{2 \chi D_{b}}{s^{3 / 2}\left(s^{1 / 2}+\chi\right)^{2}}
\end{aligned}
$$

where $k=|\mathbf{k}|=\sqrt{k_{x}^{2}+k_{y}^{2}}$. From this expression we obtain the limiting behaviors at short and long times. Thus, we observe that the short time limit $t \ll 1 / \chi^{2}$ in Laplace domain corresponds to $s \gg \chi^{2}$, such that

$$
\left\langle\mathbf{r}^{2}(s)\right\rangle_{s} \sim \frac{4 D_{s}}{s^{2}}+\frac{2 \chi D_{b}}{s^{5 / 2}} .
$$

This translates into the asymptotic time evolution

$$
\left\langle\mathbf{r}^{2}(t)\right\rangle_{s} \sim 4 D_{s} t\left(1+\frac{2}{3 \sqrt{\pi}} \frac{D_{b}}{D_{s}}\left[t \chi^{2}\right]^{1 / 2}\right) .
$$

As long as the ratio $D_{b} / D_{s}$ is sufficiently large, there is a superdiffusive component $\left\langle\mathbf{r}^{2}(t)\right\rangle_{s} \sim t^{3 / 2}$ winning over the normal surface diffusion proportional to $D_{s}$. This is exactly the famed bulk mediated superdiffusion originally obtained from scaling arguments by Bychuk and O'Shaugnessy [1, 2]. Note that this diffusional enhancement is accompanied by an almost constant number of surface particles, compare Eq. (42).

Conversely, at long times $t \gg 1 / \chi^{2}$ (or $s \ll \chi^{2}$ in Laplace domain) we find

$$
\left\langle\mathbf{r}^{2}(s)\right\rangle_{s} \sim \frac{4 D_{s}}{\chi^{2} s}+\frac{2 D_{b}}{\chi s^{3 / 2}},
$$

corresponding to the temporal behavior

$$
\left\langle\mathbf{r}^{2}(t)\right\rangle_{s} \sim \frac{4 D_{s}}{\chi^{2}}\left(1+\frac{1}{\sqrt{\pi}} \frac{D_{b}}{D_{s}}\left[t \chi^{2}\right]^{1 / 2}\right) .
$$

Somewhat surprisingly, at sufficiently long times the bulk contribution to the effective mean squared displacement dominates over the surface contribution for arbitrary ratio $D_{b} / D_{s}$, giving rise to subdiffusive behavior. This subdiffusion occurs due to the ongoing loss of surface particles into the bulk, see Eq. (43).

Instead of considering the surface mean squared displacement $\left\langle\mathbf{r}^{2}(t)\right\rangle_{s}$ we introduce the normalized effective surface mean squared displacement

$$
\left\langle\mathbf{r}^{2}(t)\right\rangle_{s}^{\text {norm }} \equiv \frac{1}{N_{s}(t)}\left\langle\mathbf{r}^{2}(t)\right\rangle_{s} .
$$

This quantity can be interpreted as the surface mean squared displacement covered by an individual particle that effectively stays on the surface and does not fully escape to the bulk. At long times this quantity has the limiting form

$$
\left\langle\mathbf{r}^{2}(t)\right\rangle_{s}^{\text {norm }} \sim 4 D_{b} t
$$

The long time diffusion corrected for the number of escaping particles displays normal diffusion, albeit with the bulk diffusivity.

In the following we neglect contributions from the surface diffusion proportional to $D_{s}$, in order not to overburden the presentation. The interesting behavior is due to the bulk mediation with weight $D_{b}$. We quantify the motion in terms of fractional order moments, before embarking for the surface propagator.

\section{Fractional order moments}

We now derive an exact expression for the $q$-th order moments $(0<q<2)$

$$
\left\langle|\mathbf{r}|^{q}(t)\right\rangle_{s}=\int|\mathbf{r}|^{q} n_{s}(\mathbf{r}, t) d^{2} \mathbf{r} .
$$

To this end we utilize the following integral on the plane:

$$
\int(1-\cos (\mathbf{k} \cdot \mathbf{r})) \frac{d k}{|\mathbf{k}|^{2+q}}=2 \pi \int_{0}^{\infty}\left(1-J_{0}(k r)\right) \frac{d k}{k^{1+q}}=2 \pi r^{q} \int_{0}^{\infty}\left(1-J_{0}(z)\right) \frac{d z}{z^{1+q}}
$$

where we used polar coordinates $r=|\mathbf{r}|$ and $k=|\mathbf{k}|$ corresponding to the two-dimensional vectors $\mathbf{r}=x \mathbf{e}_{x}+$ $y \mathbf{e}_{y}$ and $\mathbf{k}=k_{x} \mathbf{e}_{x}+k_{y} \mathbf{e}_{y}$. Thus we identify

$$
r^{q}=K(q) \int(1-\cos (\mathbf{k} \cdot \mathbf{r})) \frac{d \mathbf{k}}{k^{2+q}},
$$

with the definition

$$
K(q)=\left(2 \pi \int_{0}^{\infty}\left(1-J_{0}(z)\right) \frac{d z}{z^{1+q}}\right)^{-1}=\frac{2^{q}}{\pi^{2}} \sin \left(\frac{\pi q}{2}\right)\left[\Gamma\left(1+\frac{q}{2}\right)\right]^{2}
$$


With this trick we can rephrase the $q$ th order moment (51) as follows,

$$
\left\langle r^{q}(t)\right\rangle_{s}=K(q) \iint(1-\cos (\mathbf{k} \cdot \mathbf{r})) n_{s}(\mathbf{r}, t) \frac{d \mathbf{k}}{k^{2+q}} d \mathbf{r}=K(q) \int\left[\int n_{s}(\mathbf{r}, t) d \mathbf{r}-\int \cos (\mathbf{k} \cdot \mathbf{r}) n_{s}(\mathbf{r}, t) d \mathbf{r}\right] \frac{d \mathbf{k}}{k^{2+q}} .
$$

The integral over $d^{2} \mathbf{r}$ of the surface density is but the number of surface particles, such that

$$
\left\langle r^{q}(t)\right\rangle_{s}=K(q) \int \frac{d^{2} \mathbf{k}}{k^{2+q}}\left[N_{s}(t)-\operatorname{Re}\left\{n_{s}(\mathbf{k}, t)\right\}\right],
$$

where we replaced the Fourier cosine transform of $n_{s}(\mathbf{r}, t)$ by the real part of the exponential Fourier transform.
With the Fourier-Laplace transform (36) of the surface propagator with $D_{s}$ set to zero, for the Laplace transform of the $q$ th order moment we obtain

$$
\begin{aligned}
\left\langle r^{q}(s)\right\rangle_{s} & =K(q) \int\left[n_{s}(\mathbf{k}=0, s)-n_{s}(\mathbf{k}, s)\right] \frac{d \mathbf{k}}{k^{2+q}}=2 \pi K(q)\left[\frac{1}{s+\chi \sqrt{s}}-\frac{1}{s+\chi \sqrt{s+D_{b} k^{2}}}\right] \frac{d k}{k^{1+q}} \\
& =2 \pi K(q) \frac{\chi}{s+\chi \sqrt{s}} \int_{0}^{\infty} \frac{\sqrt{s+D_{b} k^{2}}-\sqrt{s}}{s+\chi \sqrt{s+D_{b} k^{2}}} \frac{d k}{k^{1+q}} \\
& =2 \pi K(q) \frac{\chi D_{b}}{s+\chi \sqrt{s}} \int_{0}^{\infty} \frac{d k}{\left(\sqrt{s}+\sqrt{s+D_{b} k^{2}}\right)\left(s+\chi \sqrt{s+D_{b} k^{2}}\right)} \frac{1}{k^{q-1}} \\
& =2 \pi K(q) \frac{\chi^{2}}{s+\chi \sqrt{s}}\left(\frac{D_{b}}{s}\right)^{q / 2} \int_{0}^{\infty} \frac{d y}{\left(\chi+\chi \sqrt{1+y^{2}}\right)\left(\sqrt{s}+\chi \sqrt{1+y^{2}}\right)} \frac{d y}{y^{q-1}} \\
& =2 \pi K(q) \frac{\chi D_{b}^{q / 2}}{s^{(1+q) / 2}\left(s-\chi^{2}\right)}\left\{I_{1}(q)-I_{2}(q)\right\},
\end{aligned}
$$

where on the way we introduced the substitution $y=$ $\sqrt{D_{b} / s} k$. The two integrals $I_{i}$ are defined by

$$
I_{1}(q)=\int_{0}^{\infty} \frac{y^{1-q}}{1+\sqrt{1+y^{2}}} d y
$$

and

$$
I_{2}(q)=\int_{0}^{\infty} \frac{y^{1-q}}{\sqrt{s} / \chi+\sqrt{1+y^{2}}} d y .
$$

Now we analyze the temporal behavior of the $q$ th order moment at short and long times.

\section{Long time behavior}

At long times $t \gg 1 / \chi^{2}$ (or $\left.s / \chi^{2} \ll 1\right)$ we see that $I_{1}(q)-I_{2}(q) \sim \int_{0}^{\infty}\left(\frac{1}{1+\sqrt{1+y^{2}}}-\frac{1}{\sqrt{1+y^{2}}}\right) \frac{d y}{y^{q-1}}<0$,

and

$$
\left\langle r^{q}(s)\right\rangle_{s} \simeq \frac{1}{s^{(1+q) / 2}},
$$

corresponding to the time evolution

$$
\left\langle r^{q}(t)\right\rangle_{s} \simeq t^{(q-1) / 2} .
$$

Taking normalization by the number of surface particles into account, we find

$$
\left\langle r^{q}(s)\right\rangle_{s}^{\text {norm }}=\frac{\left\langle r^{q}(s)\right\rangle_{s}}{N_{s}(t)} \simeq t^{q / 2} .
$$

That is, at long times the surface diffusion exhibits normal scaling behavior.

\section{Short time behavior}

The more interesting case is the short time behavior corresponding to the limit $t \ll 1 / \chi^{2}$ (or $\left.s / \chi^{2} \gg 1\right)$. Here we consider three separate cases:

(i) The case $1<q<2$ : Since both integrals $I_{1}$ and $I_{2}$ converge, we may simply neglect $I_{2}(q)$. Then

$$
\left\langle r^{q}(s)\right\rangle_{s} \simeq s^{-(3+q) / 2},
$$

such that after Laplace inversion we find

$$
\left\langle r^{q}(t)\right\rangle_{s} \simeq t^{(q+1) / 2} .
$$


(ii) The case $0<q<1$ : Now we should take into account both integrals,

$$
\begin{aligned}
& I_{1}(q)-I_{2}(q) \sim \\
& \frac{\sqrt{s}}{\chi} \int_{0}^{\infty} \frac{y^{1-q} d y}{\left(1+\sqrt{1+y^{2}}\right)\left(\sqrt{s} / \chi+\sqrt{1+y^{2}}\right)} .
\end{aligned}
$$

To estimate the main contribution from this difference, we split it into three parts, namely

$$
\begin{aligned}
& I_{1}(q)-I_{2}(q) \sim \\
& \frac{\sqrt{s}}{\chi}\left\{\int_{0}^{1} \ldots d y+\int_{1}^{\sqrt{s} / \chi} \ldots d y+\int_{\sqrt{s} / \chi}^{\infty} \ldots d y\right\}
\end{aligned}
$$

and evaluate each contribution separately. We find

$$
\int_{0}^{1} \ldots d y \simeq \frac{\chi}{\sqrt{s}}
$$

and then

$$
\int_{1}^{\sqrt{s} / \chi} \ldots d y \sim \int_{1}^{\sqrt{s} / \chi} \frac{1}{y \sqrt{s} / \chi} \frac{d y}{y^{q-1}} \sim\left(\frac{\chi}{\sqrt{s}}\right)^{q}>\frac{\chi}{s} .
$$

Finally,

$$
\int_{\sqrt{s} / \chi}^{\infty} \frac{d y}{y^{1+q}} \simeq\left(\frac{\chi}{\sqrt{s}}\right)^{q}
$$

Thus the main contribution come from Eqs. (68b) and (68c), and

$$
I_{1}(q)-I_{2}(q) \simeq s^{(1-q) / 2} .
$$

With this estimate the Laplace transform of the $q$ th order moment, Eq. (57), has the leading order behavior

$$
\left\langle r^{q}(s)\right\rangle_{s} \simeq \frac{s^{(1-q) / 2}}{s^{(3+q) / 2}} \simeq \frac{1}{s^{1+q}} .
$$

This corresponds to

$$
\left\langle r^{q}(t)\right\rangle_{s} \simeq t^{q}
$$

in the time domain.

(iii) The case $q=1$ : This special case requires some care. We start with the substitution $y=\tan \phi$ in Eqs. (58) and (59). Then the integrals $I_{i}$ become

$$
I_{1}(1)=\int_{0}^{\pi / 2} \frac{d \phi}{\cos \phi(1+\cos \phi)},
$$

and

$$
I_{2}(1)=\int_{0}^{\pi / 2} \frac{d \phi}{\cos \phi(1+\sqrt{s} \cos \phi / \chi)} .
$$

We can now rewrite Eq. (57) in the form

$$
\begin{aligned}
\langle r(s)\rangle_{s} & =2 \pi K(1) \frac{\chi D_{b}^{1 / 2}}{s\left(s-\chi^{2}\right)} \\
& \times\left(\frac{\sqrt{s}}{\chi} \int_{0}^{\pi / 2} \frac{d \phi}{1+\sqrt{s} \cos \phi / \chi}-1\right),
\end{aligned}
$$

where we used [13]

$$
\int_{0}^{\pi / 2} \frac{d \phi}{1+\cos \phi}=1
$$

For $s / \chi^{2}<1$ the integral in the parenthesis of Eq. (74) becomes 13 ]

$$
\int_{0}^{\pi / 2} \frac{d \phi}{1+\sqrt{s} \cos \phi / \chi}=\frac{2}{\sqrt{1-s / \chi^{2}}} \arctan \sqrt{\frac{1-\sqrt{s} / \chi}{1+\sqrt{s} / \chi}} .
$$

Thus, for Eq. (74) we find

$$
\begin{aligned}
& \langle r(s)\rangle_{s}=2 \pi K(1) \frac{\chi D_{b}^{1 / 2}}{s\left(s-\chi^{2}\right)} \\
& \times\left(\frac{2 \sqrt{s}}{\chi} \frac{1}{\sqrt{1-s / \chi^{2}}} \arctan \sqrt{\frac{1-\sqrt{s} / \chi}{1+\sqrt{s} / \chi}}-1\right),
\end{aligned}
$$

so that we find the $s \rightarrow 0$ behavior

$$
\langle r(s)\rangle_{s} \sim \frac{D_{b}^{1 / 2}}{\chi s} .
$$

After Laplace inversion,

$$
\langle r(t)\rangle_{s} \sim \frac{D_{b}^{1 / 2}}{\chi}, \text { and }\langle r(t)\rangle_{s}^{\text {norm }} \sim\left(D_{b} t\right)^{1 / 2}
$$

at long times, $t \gg 1 / \chi^{2}$, consistent with Eq. (63). Conversely, for $s / \chi^{2}>1$ we employ [13]

$$
\begin{aligned}
& \int_{0}^{\pi / 2} \frac{d \phi}{1+\sqrt{s} \cos \phi / \chi}=\frac{1}{\sqrt{s / \chi^{2}-1}} \\
& \times \ln \frac{\sqrt{s / \chi^{2}-1}+\sqrt{s} / \chi-1}{\sqrt{s / \chi^{2}-1}+1-\sqrt{s} / \chi} .
\end{aligned}
$$

Thus we find

$$
\langle r(s)\rangle_{s}=2 \pi K(1) \frac{\chi D_{b}^{1 / 2}}{s\left(s-\chi^{2}\right)}\left[\frac{\sqrt{s} / \chi}{\sqrt{s / \chi^{2}-1}} \ln \left(\frac{2 \sqrt{s}}{\chi}\right)-1\right] .
$$

We thus obtain the limiting form at large $s$

$$
\langle r(s)\rangle_{s} \sim \frac{\chi D_{b}^{1 / 2}}{s^{2}} \ln \left(\frac{2 \sqrt{s}}{\chi}\right) .
$$

Back-transformed this results in

$$
\langle r(t)\rangle_{s} \approx\left\langle r^{q}(s)\right\rangle_{s}^{\text {norm }} \sim t \ln t
$$

at short times $t \ll 1 / \chi^{2}$.

Summarizing our results for $q$ th order moments, at short times $t \ll 1 / \chi^{2}$ the effective surface diffusion exhibits anomalous scaling: the $q$ th order moment of the radius scales like $t^{q}$ for $0<q<1$ and $t^{(q+1) / 2}$ for $1<q<2$, while the first moment includes a logarithmic contribution, $t \ln t$, consistent with the earlier results in Ref. [2]. At long times $t \gg 1 / \chi^{2}$ the $q$ th order moments scale normally with time, proportional to $t^{q / 2}$. 


\section{SURFACE PROPAGATOR}

We now turn to the behavior of the surface propagator. If we neglect surface diffusion (i.e., $D_{s}=0$ ) the surface propagator from Eq. (36) becomes

$$
n_{s}(\mathbf{k}, s)=\frac{1}{s+\chi \sqrt{s+D_{b} k^{2}}} .
$$

We perform an inverse Laplace transformation along the Bromwich path:

$$
\begin{aligned}
& n_{s}(\mathbf{k}, t)=\int_{\mathrm{Br}} \frac{\exp (s t)}{s+\chi \sqrt{s+D_{b} k^{2}}} \frac{d s}{2 \pi i}=\int_{\mathrm{Br}} \frac{\exp \left(s \chi^{2} t\right)}{s+\sqrt{s+\kappa^{2}}} \frac{d s}{2 \pi i}=e^{-\kappa^{2} \chi^{2} t} \int_{\mathrm{Br}} \frac{\exp \left(s \chi^{2} t\right)}{s+\sqrt{s}-\kappa^{2}} \frac{d s}{2 \pi i} \\
& =e^{-\kappa^{2} \chi^{2} t} \int_{\operatorname{Br}} \frac{\exp \left(s \chi^{2} t\right)}{\left(\sqrt{s}+\frac{1}{2}+\sqrt{\kappa^{2}+\frac{1}{4}}\right)\left(\sqrt{s}+\frac{1}{2}-\sqrt{\kappa^{2}+\frac{1}{4}}\right)} \frac{d s}{2 \pi i} \\
& =\frac{\exp \left(-\kappa^{2} \chi^{2} t\right)}{2 \sqrt{\kappa^{2}+1 / 4}}\left(\int_{\mathrm{Br}} \frac{\exp \left(s \chi^{2} t\right)}{\sqrt{s}+\frac{1}{2}-\sqrt{\kappa^{2}+\frac{1}{4}}} \frac{d s}{2 \pi i}-\int_{\mathrm{Br}} \frac{\exp \left(s \chi^{2} t\right)}{\left.\sqrt{s}+\frac{1}{2}+\sqrt{\kappa^{2}+\frac{1}{4}} \frac{d s}{2 \pi i}\right)}\right. \\
& =\frac{\exp \left(-\kappa^{2} \chi^{2} t\right)}{2 \sqrt{\kappa^{2}+1 / 4}}\left(\mathscr{L}^{-1}\left\{\frac{1}{\sqrt{s}-b} ; s \rightarrow t^{\prime}\right\}_{b=\sqrt{\kappa^{2}+1 / 4}-1 / 2, t^{\prime}=t \chi^{2}}-\mathscr{L}^{-1}\left\{\frac{1}{\sqrt{s}+a} ; s \rightarrow t^{\prime}\right\}_{a=1 / 2+\sqrt{\kappa^{2}+1 / 4}, t^{\prime}=t \chi^{2}}\right)(85)
\end{aligned}
$$

where on the way we introduced the substitutions $s^{\prime}=s / \chi^{2}$ and $\kappa^{2}=D_{b} k^{2} / \chi^{2}$. With

$$
\mathscr{L}^{-1}\left\{\frac{1}{\sqrt{s}+a} ; s \rightarrow t\right\}=\frac{1}{\sqrt{\pi t}}-a e^{a^{2} t} \operatorname{erfc}(a \sqrt{t})
$$

we finally arrive at the Fourier transform of the surface propagator,

$$
\begin{aligned}
n_{s}(\mathbf{k}, t)= & \frac{1}{2 \sqrt{\kappa^{2}+1 / 4}}\left\{\left[\frac{1}{2}+\sqrt{\kappa^{2}+\frac{1}{4}}\right] \exp \left(\left[\frac{1}{2}+\sqrt{\kappa^{2}+\frac{1}{4}}\right] t \chi^{2}\right) \operatorname{erfc}\left(\left[\frac{1}{2}+\sqrt{\kappa^{2}+\frac{1}{4}}\right] \chi \sqrt{t}\right)\right. \\
& \left.+\left(\sqrt{\kappa^{2}+\frac{1}{4}}-\frac{1}{2}\right) \exp \left(\left[\frac{1}{2}-\sqrt{\kappa^{2}+\frac{1}{4}}\right] t \chi^{2}\right) \operatorname{erfc}\left(\left[\frac{1}{2}-\sqrt{\kappa^{2}+\frac{1}{4}}\right] \chi \sqrt{t}\right)\right\} .
\end{aligned}
$$

In compact form,

$$
\begin{aligned}
n_{s}(\mathbf{k}, t)= & \frac{1}{\alpha+\beta}\left\{\alpha e^{\alpha \chi^{2} t} \operatorname{erfc}(\alpha \chi \sqrt{t})\right. \\
& \left.+\beta e^{-\beta \chi^{2} t} \operatorname{erfc}(-\beta \chi \sqrt{t})\right\}
\end{aligned}
$$

with

$$
\alpha=\sqrt{\kappa^{2}+\frac{1}{4}}+\frac{1}{2}, \quad \beta=\sqrt{\kappa^{2}+\frac{1}{4}}-\frac{1}{2} .
$$

We now calculate the surface propagator $n_{s}(\mathbf{r}, t)$ in the limits of short and long times.

\section{A. Short time behavior}

At short times $t \ll \chi^{-2}$ we distinguish between the central part of the propagator and its wings. Starting with the central part, we thus focus on distances $r \ll$ $\left(D_{b} t\right)^{1 / 2}$. Due to $\kappa^{2}=D_{b} k^{2} / \chi^{2}$, this means that we consider $\kappa \chi \sqrt{t} \gg 1$, and therefore $\kappa^{2} \gg 1$. Consequently we find $\alpha \approx \beta \approx \kappa, \alpha \chi \sqrt{t} \approx \beta \chi \sqrt{t} \approx \kappa \chi \sqrt{t} \gg 1$, and thus

$$
\operatorname{erfc}(\alpha \chi \sqrt{t}) \sim \frac{\exp \left(-\alpha^{2} \chi^{2} t\right)}{\sqrt{\pi} \alpha \chi \sqrt{t}} .
$$

Then, the first term in the curly brackets of Eq. (88) is (note that $\alpha^{2}-\alpha=\kappa^{2}$ )

$$
\alpha e^{\alpha \chi^{2} t} \operatorname{erfc}(\alpha \chi \sqrt{t}) \approx \frac{1}{\sqrt{\pi \chi^{2} t}} e^{-\kappa^{2} \chi^{2} t}
$$

The error function in the second term of Eq. (88) is approximately 2 . Thus, the second term in the curly brackets of Eq. (88) prevails, and we obtain

$$
n_{s}(\mathbf{k}, t) \sim \exp \left(-\kappa \chi^{2} t\right) .
$$


The Fourier inversion is accomplished with the help of the integral [13]

$$
\int_{0}^{\infty} x e^{-p x} J_{0}(c x) d x=\frac{p}{\left(p^{2}+c^{2}\right)^{3 / 2}},
$$

and we obtain

$$
\begin{aligned}
n_{s}(\mathbf{r}, t) & \sim \int e^{-i \mathbf{k} \cdot \mathbf{r}} n_{s}(\mathbf{k}, t) \frac{d \mathbf{k}}{4 \pi^{2}} \\
& \sim \int_{0}^{\infty} e^{-\chi \sqrt{D_{b}} k t} J_{0}(k r) \frac{k d k}{2 \pi}
\end{aligned}
$$

such that

$$
n_{s}(\mathbf{r}, t) \sim \frac{\chi D_{b}^{1 / 2} t}{2 \pi\left(r^{2}+\chi^{2} D_{b} t^{2}\right)^{3 / 2}} .
$$

This is exactly the two-dimensional Cauchy distribution obtained by Bychuk and O'Shaugnessy from scaling arguments [1]. We see that indeed radius and time are coupled linearly in superdiffusive fashion, $r \sim t$. However, as we will see this Cauchy form holds only for the central part of the surface propagator. It assumes steeper wings such that at all times any moment of the propagator exists. We are quantifying these wings below.

The number of particles populating this cental Cauchy domain of the propagator, i.e., within a circle of radius $\left(D_{b} t\right)^{1 / 2}$ is

$$
\int^{\left(D_{b} t\right)^{1 / 2}} n_{s}(\mathbf{r}, t) d \mathbf{r} \sim 1-\chi \sqrt{t},
$$

i.e., at sufficiently short times a major portion of the particles are contained in the Cauchy domain. Indeed, these particles show the superdiffusive mean squared displacement

$$
\left\langle\mathbf{r}^{2}\right\rangle_{s}=\int^{\left(D_{b} t\right)^{1 / 2}} \mathbf{r}^{2} n_{s}(\mathbf{r}, t) d \mathbf{r} \approx \chi D_{b} t^{3 / 2},
$$

consistent with result (46) for $D_{s}=0$, the prefactor differing by approximately a factor $3 / 2$.

The outer part of the propagator at short times is found for $r \gg\left(D_{b} t\right)^{1 / 2}$. This region can be divided into two subregions, namely, $\kappa^{2} \gg 1$ corresponding to $D_{b} t \ll r^{2} \ll D_{b} / \chi^{2}$, and $\kappa^{2} \ll 1$ corresponding to $r^{2} \gg D_{b} / \chi^{2}$. However, it is easy to check that in both of these sub-regions we have $\alpha \chi \sqrt{t} \ll 1$ and $\beta \chi \sqrt{t} \ll 1$, due to the combination of the conditions of short times $(\chi \sqrt{t} \ll 1)$ and large radii $(\kappa \chi \sqrt{t} \ll 1)$. Thus both error functions in Eq. (88) are expanded at small values of their arguments,

$$
\operatorname{erfc}( \pm z) \sim 1 \mp \frac{2}{\sqrt{\pi}} z \pm \frac{2}{3 \sqrt{\pi}} z^{3}+\ldots,
$$

with $z=\alpha \chi \sqrt{t}<1$ and $z=\beta \chi \sqrt{t}<1$, respectively. Our result is

$$
n_{s}(\mathbf{k}, t) \sim 1-\frac{2}{\sqrt{\pi}} \chi \sqrt{t}-\frac{2}{3 \sqrt{\pi}} k^{2} D_{b} \chi t^{3 / 2}
$$

plus terms of order $k^{4}$ and higher. We conclude that at the wings the central Cauchy distribution is truncated by Gaussian tails whose dispersion grows like $t^{3 / 2}$.

\section{B. Long time behavior}

This is the limit $t \gg 1 / \chi^{2}$. As this implies $\alpha \chi \sqrt{t} \gg 1$ we may expand the error function as

$$
\operatorname{erfc}(\alpha \chi \sqrt{t}) \sim \frac{\exp \left(-\alpha^{2} \chi^{2} t\right)}{\sqrt{\pi} \alpha \chi \sqrt{t}} .
$$

We consider the shape of the propagator in the main part excluding the region near the origin, i.e, we have

$$
r^{2} \gg D_{b} \sqrt{t} / \chi \gg D_{b} / \chi^{2}
$$

In this domain we may use $\kappa \ll 1, \alpha \approx 1$, and $\kappa^{2} \chi \sqrt{t} \ll$ 1. Since $\beta=\sqrt{\kappa^{2}+1 / 4}-1 / 2 \approx \kappa^{2}$, we have $\beta \chi \sqrt{t} \ll 1$, and thus the second error function becomes

$$
\operatorname{erfc}(-\beta \chi \sqrt{t}) \approx 1 .
$$

The surface propagator thus assumes the shape (recall that $\alpha^{2}-\alpha=\kappa^{2}$ )

$$
\begin{aligned}
n_{s}(\mathbf{k}, t) & \sim \frac{1}{\alpha}\left(\alpha e^{\alpha \chi^{2} t} \frac{\exp \left(-\alpha^{2} \chi^{2} t\right)}{\sqrt{\pi} \alpha \chi \sqrt{t}}+\kappa^{2} \exp \left(-\beta \chi^{2} t\right)\right) \\
& \sim \frac{\exp \left(-\kappa^{2} \chi^{2} t\right)}{\sqrt{\pi} \chi \sqrt{t}}+\kappa^{2} e^{-\kappa^{2} \chi^{2} t} \\
& \sim \frac{\exp \left(-\kappa^{2} \chi^{2} t\right)}{\sqrt{\pi} \chi \sqrt{t}} .
\end{aligned}
$$

Equivalently, this means that

$$
n_{s}(\mathbf{k}, t) \sim \frac{\exp \left(-k^{2} D_{b} t\right)}{\sqrt{\pi} \chi \sqrt{t}} .
$$

Inverse Fourier transform produces the propagator at long times,

$$
\begin{aligned}
n_{s}(\mathbf{r}, t) & \sim \int e^{-i \mathbf{k} \cdot \mathbf{r}} n_{s}(\mathbf{k}, t) \frac{d \mathbf{k}}{4 \pi^{2}} \\
& \sim \frac{1}{\chi \sqrt{\pi t}} \int_{0}^{\infty} J_{0}(k r) e^{-k^{2} D_{b} t} \frac{k d k}{2 \pi} .
\end{aligned}
$$

From this we obtain the Gaussian form

$$
n_{s}(\mathbf{r}, t) \sim \frac{1}{\chi \sqrt{\pi t}} \frac{\exp \left(-r^{2} /\left[4 D_{b} t\right]\right)}{4 \pi D_{b} t} .
$$

The number of particles contained in this part of the long-time propagator follows from

$$
\int_{r^{2} \geq D_{b} \sqrt{t} / \chi} n_{s}(\mathbf{r}, t) d \mathbf{r} \sim \frac{1}{\chi \sqrt{\pi t}} .
$$

Comparison to Eq. (43) shows that these are virtually all particles still on the surface. Thus the Gaussian (106) 
indeed dominates the behavior at long times. This statement is also corroborated from calculation of the surface mean squared displacement from Eq. (106) in the domain $r^{2} \geq D_{b} \sqrt{t} / \chi$

$$
\begin{aligned}
\left\langle\mathbf{r}^{2}\right\rangle_{s} & \sim \int_{r^{2} \geq D_{b} \sqrt{t} / \chi} r^{2} n_{s}(\mathbf{r}, t) d \mathbf{r} \\
& \sim \frac{4 D_{b} t}{\chi \sqrt{\pi t}}\left[1+o\left(\frac{1}{t \chi^{2}}\right)\right] .
\end{aligned}
$$

Again, up to a small correction, this term coincides with our exact result for the long time behavior, Eq. (48).

\section{CONCLUSIONS}

Complementing our earlier studies of bulk mediated surface diffusion on a cylinder [10], we presented a strictly analytical treatment of the bulk mediated surface diffusion problem on a flat surface. While our analysis corroborates previous scaling results of superdiffusion and the Cauchy form of the surface propagator, we find corrections to this behavior sufficiently far away from the origin, and at long times. Thus, the Cauchy domain is rectified by a Gaussian decay of the extremities of the propagator which we derive explicitly. As a consequence finite spatial moments of any order remain finite. At long times a Gaussian regime takes over, assembling virtually all particles.
Our analysis is based on the exact representation of the surface propagator in Fourier-Laplace space from which systematic expansions are obtained. For the number of surface particles we find an initial stagnation, followed at longer times by a decrease proportional to $1 / \sqrt{t}$. The mean squared displacement $\left\langle\mathbf{r}^{2}\right\rangle_{s}$ turns from the initial superdiffusive behavior proportional to $t^{3 / 2}$ to a subdiffusive form $\left\langle\mathbf{r}^{2}\right\rangle_{s} \simeq t^{1 / 2}$. The latter corresponds to normal diffusion, albeit with the bulk diffusivity, if normalized to the decay of the surface particles. Our analysis is complemented with the derivation of fractional order moments for the surface propagation.

\section{Acknowledgments}

RM thanks the Academy of Finland for financial support within the FiDiPro scheme.

\section{Appendix A: Alternative derivation of the surface density}

In this appendix we derive the Lévy flight behavior recovered in the main part of the text based on a complementary approach developed in Refs. $14-16$. We start with the coupled diffusion equation along the surface (equivalent to Eq. (13)) as

$$
\frac{\partial n_{s}}{\partial t}=D_{s}\left(\frac{\partial^{2}}{\partial x^{2}}+\frac{\partial^{2}}{\partial y^{2}}\right) n_{s}-\frac{1}{\tau_{\mathrm{des}}} n_{s}+\frac{1}{\tau_{\mathrm{des}}} \int d x^{\prime} \int d y^{\prime} \int_{0}^{t} d t^{\prime} W_{\mathrm{bulk}}\left(x-x^{\prime}, y-y^{\prime}, t-t^{\prime}\right) n_{s}\left(x^{\prime}, y^{\prime}, t^{\prime}\right)
$$

where the last term represents rebinding events of particles that previously desorbed from the surface. The kernel $W_{\text {bulk }}(x, y, t)$ in the convolution is the probability density to attach at $(x, y)$ at time $t$ after desorption from the origin at time zero. Together, the last two terms of Eq. (A1) represent jumps along the surface mediated by the bulk. Note that we have implicitly assumed that all particles start at the surface at $t=0$. Otherwise an additional term would be needed to represent particles binding time to the surface for the first time.

To obtain $W_{\text {bulk }}$ we need to solve the bulk diffusion equation, Eq. (12), with a single particle released from the boundary at the origin at time zero. This can be achieved by choosing a boundary condition

$$
\left.D_{b} \frac{\partial n_{b}}{\partial z}\right|_{z=0}=\left.\frac{1}{\mu \tau_{\mathrm{des}}} n_{b}\right|_{z=0}-\delta(x) \delta(y) \delta(t)
$$

This equation corresponds to the current of particles into the surface, the second term on the right hand side representing the initial release. Supplementing this by boundary conditions of vanishing density at infinity, $n_{b} \rightarrow 0$ as $|x|,|y|, z \rightarrow \infty$, and the initial condition of finding no particles in the bulk, $n_{b}(x, y, z, t=0)=0$, we obtain $W_{\text {bulk }}$ from the solution in terms of the flux into the boundary after the initial release: $W_{\text {bulk }}(x, y, t)=$ $n_{b}(x, y, z=0, t) /\left(\mu \tau_{\text {des }}\right)$.

To solve Eq. (12) we again Fourier transform along $x$ and $y\left(x \rightarrow k_{x}\right.$ and $\left.y \rightarrow k_{y}\right)$ and Laplace transform in time $(t \rightarrow s)$. Together with the initial condition and the boundary conditions at infinity it is easily found that

$$
n_{b}\left(k_{x}, k_{y}, z, s\right)=e^{-z \sqrt{k^{2}+s / D_{b}}} n_{b}\left(k_{x}, k_{y}, z=0, s\right)
$$

where $k^{2}=k_{x}^{2}+k_{y}^{2}$. Insertion of Eq. (A33) into Eq. (A2) leads to a bulk density at the boundary which is

$$
n_{b}\left(k_{x}, k_{y}, z=0, s\right)=\frac{1}{\left(\mu \tau_{\mathrm{des}}\right)^{-1}+D_{b} \sqrt{k^{2}+s / D_{b}}}
$$


Multiplication by the rate constant $1 /\left(\mu \tau_{\text {des }}\right)$ then leads to

$$
W_{\mathrm{bulk}}\left(k_{x}, k_{y}, s\right)=\frac{1}{1+\mu \tau_{\mathrm{des}} D_{b} \sqrt{k^{2}+s / D_{b}}} .
$$

We are now ready to obtain the surface density $n_{s}$ in Fourier-Laplace space. From Eq. (A1) with the initial condition $n_{s}(x, y, t=0)=n_{0} \delta(x) \delta(y)$ we obtain

$$
n_{s}\left(k_{x}, k_{y}, s\right)=\frac{n_{0}}{s+D_{s} k^{2}+\tau_{\mathrm{des}}^{-1}\left[1-W_{\mathrm{bulk}}\left(k_{x}, k_{y}, s\right)\right]},
$$

in which Eq. A5 can be directly inserted. To obtain the expression in Eq. (36) of the main text one takes the limit of the desorption rate constant $\tau_{\text {des }}^{-1}$ and adsorption rate constant $1 /\left(\mu \tau_{\text {des }}\right)$ going to infinity while keeping their ratio $\mu$ fixed, which leads to

$$
\tau_{\mathrm{des}}^{-1}\left[1-W_{\mathrm{bulk}}\left(k_{x}, k_{y}, s\right)\right] \rightarrow \mu D_{b} \sqrt{k^{2}+s / D_{b}}
$$

Together with $\chi=\mu \sqrt{D_{b}}$ and $n_{0}=1$ this makes Eq. (A6) equivalent to Eq. (36).

As a byproduct of the approach of this appendix we remark that we can now obtain the distribution of bulk mediated jump lengths. These are given by $\lambda(r=$ $\left.\sqrt{x^{2}+y^{2}}\right)=2 \pi r W_{\text {bulk }}(x, y, s=0)$. From Eq. A5 we find [performing the angular part of the inverse Fourier transform similarly to Eq. (95)]

$$
\lambda(r)=2 \pi r \int_{0}^{\infty} \frac{k d k}{2 \pi} \frac{J_{0}(k r)}{1+\mu \tau_{\mathrm{des}} D_{b} k}=\frac{1}{\mu \tau_{\mathrm{des}} D_{b}}-\frac{\pi r}{2\left(\mu \tau_{\mathrm{des}} D_{b}\right)^{2}}\left[\mathbf{H}_{0}\left(\frac{r}{\mu \tau_{\mathrm{des}} D_{b}}\right)-Y_{0}\left(\frac{r}{\mu \tau_{\mathrm{des}} D_{b}}\right)\right]
$$

where $\mathbf{H}_{\nu}$ are the Struve functions and $Y_{\nu}$ are the Bessel functions of the second kind. If we expand $\lambda(r)$ asymptotically at large $r$ we obtain a power law

$$
\lambda(r) \sim \frac{\mu \tau_{\mathrm{des}} D_{b}}{r^{2}}
$$

Thus we see that the Cauchy propagator (95), arising for $D_{s}=0$ in the short time regime where almost all particles are still bound on the surface, is connected with a Lévy flight behavior based on a power law jump length distribution.

\section{Appendix B: Derivation of the coupling equation (15)}

We start by rewriting Eq. (30) in the form

$$
n_{b}(x, y, z, t)=\frac{\mu z}{\left(4 \pi D_{b}\right)^{3 / 2}} \int_{0}^{t} d \tau \int d x^{\prime} \int d y^{\prime} \frac{n_{s}\left(x^{\prime}, y^{\prime}, t-\tau\right)}{\tau^{5 / 2}} \exp \left(-\frac{\left(x-x^{\prime}\right)^{2}+\left(y-y^{\prime}\right)^{2}+z^{2}}{4 D_{b} \tau}\right) .
$$

We know that the Gaussian is a limiting representation of the $\delta$ function:

$$
\lim _{\tau \rightarrow 0} \frac{1}{\sqrt{4 \pi D_{b} \tau}} \exp \left(-\frac{\left(x-x^{\prime}\right)^{2}}{4 D_{b} \tau}\right)=\delta\left(x-x^{\prime}\right) .
$$

Let us now introduce the new variable $\tau^{\prime}=\tau / z^{2}$, such that relation (B1) turns into

$$
n_{b}(x, y, z, t)=\frac{\mu z}{\left(4 \pi D_{b}\right)^{3 / 2}} \int_{0}^{t / z^{2}} z^{2} d \tau^{\prime} \int d x^{\prime} \int d y^{\prime} \frac{n_{s}\left(x^{\prime}, y^{\prime}, t-\tau^{\prime} z^{2}\right)}{z^{5}\left(\tau^{\prime}\right)^{5 / 2}} \exp \left(-\frac{\left(x-x^{\prime}\right)^{2}}{4 D_{b} \tau^{\prime} z^{2}}-\frac{\left(y-y^{\prime}\right)^{2}}{4 D_{b} \tau^{\prime} z^{2}}-\frac{z^{2}}{4 D_{b} \tau^{\prime} z^{2}}\right)
$$

Rearranging terms,

$$
n_{b}(x, y, z, t)=\int_{0}^{t / z^{2}} d \tau^{\prime} \int d x^{\prime} \int d y^{\prime} \frac{\mu n_{s}\left(x^{\prime}, y^{\prime}, t-\tau^{\prime} z^{2}\right)}{\left(\tau^{\prime}\right)^{3 / 2}} \frac{\exp \left(-\frac{\left(x-x^{\prime}\right)^{2}}{4 D_{b} \tau^{\prime} z^{2}}\right)}{\sqrt{4 \pi D_{b} \tau^{\prime} z^{2}}} \frac{\exp \left(-\frac{\left(y-y^{\prime}\right)^{2}}{4 D_{b} \tau^{\prime} z^{2}}\right)}{\sqrt{4 \pi D_{b} \tau^{\prime} z^{2}}} \frac{\exp \left(-\frac{1}{4 D_{b} \tau^{\prime}}\right)}{\sqrt{4 \pi D_{b}}} .
$$

We are now in the position to take the limit $z \rightarrow 0$, producing (we omit the prime in $\tau^{\prime}$ )

$$
\lim _{z \rightarrow 0} n_{b}(x, y, z, t)=\int_{0}^{\infty} d \tau \int d x^{\prime} \int d y^{\prime} \frac{\mu n_{s}\left(x^{\prime}, y^{\prime}, t\right)}{\tau^{3 / 2}} \delta\left(x-x^{\prime}\right) \delta\left(y-y^{\prime}\right) \frac{\exp \left(-\frac{1}{4 D_{b} \tau}\right)}{\sqrt{4 \pi D_{b}}},
$$


which directly reduces to the expression

$$
\lim _{z \rightarrow 0} n_{b}(x, y, z, t)=\mu n_{s}(x, y, t) \frac{1}{\sqrt{4 \pi D_{b}}} \int_{0}^{\infty} d \tau \frac{\exp \left(-\frac{1}{4 D_{b} \tau}\right)}{\tau^{3 / 2}}
$$

Finally, with the substitution $\zeta=\tau^{-1 / 2}$ with the Jacobian $d \zeta=-1 /\left(2 \tau^{3 / 2}\right)$, the remaining integral represents the normalization of a Gaussian,

$$
\lim _{z \rightarrow 0} n_{b}(x, y, z, t)=\mu n_{s}(x, y, t) \frac{2}{\sqrt{4 \pi D_{b}}} \int_{0}^{\infty} d \zeta \exp \left(-\frac{\zeta^{2}}{4 D_{b}}\right)=\mu n_{s}(x, y, t),
$$

and the equivalence with the coupling equation (15) is established.

[1] O. V. Bychuk and B. O'Shaughnessy, Phys. Rev. Lett. 74, 1795 (1995).

[2] O. V. Bychuk and B. O'Shaugnessy, J. Chem. Phys. 101, 772 (1994).

[3] R. Valiullin, R. Kimmich, and N. Fatkullin, Phys. Rev. E 56, 4371 (1997).

[4] J. A. Revelli, C. E. Budde, D. Prato, and H. S. Wio, New J. Phys. 7, 16 (2005).

[5] S. Stapf, R. Kimmich and R.-O. Seitter, Phys. Rev. Lett. 75, 2855 (1995).

[6] Y. Georgievskii, E. S. Medvedev, and A. A. Stuchebrukhov, J. Chem. Phys. 116, 1692 (2002).

[7] O. Bénichou, D. Grebenkov, P. Levitz, C. Loverdo, and R. Voituriez, Phys. Rev. Lett. 105, 150606 (2010); J. Stat. Phys. 142, 657 (2011).

[8] M. E. Toimil-Molares, L. Röntzsch, W. Sigle, K.-H. Heinig, C. Trautmann, and R. Neumann, Adv. Functional Mat. 22, 695 (2012).

[9] R. Metzler and J. Klafter, Phys. Rep. 339, 1 (2000); J. Phys. A 37, R161 (2004).

[10] A. V. Chechkin, I. M. Zaid, M. A. Lomholt, I. M. Sokolov, and R. Metzler, Phys. Rev. E 79, 040105(R) (2009); J. Chem. Phys. 134, 204116 (2011).

[11] H. S. Carslaw and J. C. Jaeger, Conduction of heat in solids, 2nd edition (Clarendon Press, Oxford, UK, 1959), chapter 2; A. N. Tikhonov and A. A. Samarskii, Equations of mathematical physics (Pergamon Press, Oxford, UK, 1963), chapter 3.

[12] M. Abramowitz and I. A. Stegun, Handbook of mathematical functions (National Bureau of Standards, Washington, DC, 1972).

[13] A. B. Prudnikov, Yu. A. Brychkov and O. I. Marichev, Integrals and Series (Gordon and Breach Science Publishers, 1992).

[14] M. A. Lomholt, I. M. Zaid, and R. Metzler, Phys. Rev. Lett. 98, 200603 (2007).

[15] I. M. Zaid, M. A. Lomholt, and R. Metzler, Biophys. J. 97, 710 (2009).

[16] M. A. Lomholt, B. van den Broek, S.-M. J. Kalisch, G. J. L. Wuite, and R. Metzler, Proc. Natl. Acad. Sci. USA 106, 8204 (2009). 